\title{
Modèle d'un système intégré inno- vant pour hôpitaux de soins aigus
}

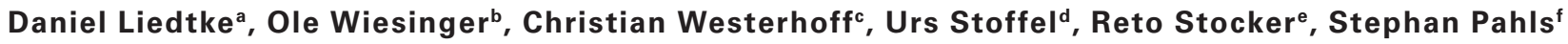 \\ ${ }^{a}$ Dr, COO Groupe de cliniques privées Hirslanden; ${ }^{b}$ Dr med., CEO Groupe de cliniques privées Hirslanden; ${ }^{c}$ Dr med., CCO Groupe de cliniques privées \\ Hirslanden; ${ }^{d}$ Dr med., Cabinet Chirurgical Enge, Zurich; ${ }^{e}$ Prof. Dr med., Institut d'anesthésiologie et de soins intensifs Klinik Hirslanden, Zurich; \\ ${ }^{\mathrm{f}}$ Dr med., Pahls Consulting, Zurich
}

A la suite de la nouvelle planification et du nouveau financement hospitalier, le paysage hospitalier suisse vit une mutation de grande ampleur. Les cantons prescrivent en détail aux hôpitaux les prestations qu'ils peuvent fournir, les exigences à respecter en matière de qualité, la quantité de personnel à former et les coûts acceptables par cas. Les hôpitaux sont en concurrence sur le front des patients, du personnel de soin et des meilleurs médecins, mais ils sont en même temps restreints par la pénurie en personnel qualifié, la pression exercée par les coûts et la marge de manœuvre des listes hospitalières. De nombreux hôpitaux réagissent à ces défis par un changement de forme juridique, la mise en place d'un lean management, le renouvellement de l'infrastructure hospitalière, la constitution de centres de compétence et le renforcement de coopérations. Est-ce suffisant? D’après nous, deux sujets importants sont négligés: l'organisation du corps médical et l'attribution des tâches de ce dernier. Ces sujets sont essentiels pour tous les hôpitaux et ont une influence sur l'importance et la pérennité de la compétitivité.

\section{Revenir à une répartition des tâches}

Pour certains, il s'agit de revenir à une répartition intelligente des tâches en hôpital. Les tâches non médicales des cadres médicaux ont constamment augmenté ces dernières années. Elles diminuent l'attractivité du lieu de travail, ne sont pas efficientes en termes de coût et ne sont plus acceptables à long terme du fait du manque de personnel qualifié. Pour d'autres, il s'agit de mettre en place une organisation fonctionnelle performante pour le corps médical en hôpital. Ces dernières années, deux profils d'activité hospitalière différents se sont dégagés. D'un côté, les fonctions transdisciplinaires médicales, comme par ex. l'anesthésie et la médecine intensive, la médecine interne générale et la médecine d'urgence, ou bien encore la radiologie, qui doivent se comporter en hôpital comme des prestataires médicaux ou des «médecins de premier recours». De l'autre, des équipes médicales sous- ou «super»-spécialisées toujours plus nombreuses qui s'occupent des besoins spécifiques des médecins référents et des patients. Cela montre que ces différentes tâches médicales nécessitent également une organisation et une direction différentes.

Bien que Porter et Teisberg aient décrit leur modèle des «unités de pratique intégrées» (UPI) [1] il y a bientôt 10 ans, l'organisation du corps médical dans de nombreux hôpitaux persiste à suivre les modèles traditionnels. Comment améliorer la relation entre hôpitaux et médecins? Professeur d'économie à Zurich, Tilman

\section{L'organisation du corps médical et l'attribution} des tâches de ce dernier sont essentielles.

Slembeck a été cité brièvement à ce sujet dans le SonntagsBlick [2], avec une théorie digne d'attention. D'après Slembeck, les hôpitaux sont avant tout devenus des centres logistiques, qui mettent à disposition des équipements et des salles de consultation et d'opération. Les opérations sont réalisées par des médecins indépendants organisés en réseaux, comme dans le système des médecins accrédités. Par conséquent, d'ici 10 à 15 ans, il ne devrait plus avoir de chirurgiens en poste que dans les hôpitaux universitaires. Cette théorie est audacieuse, mais pas tout à fait aberrante, car nous connaissons déjà des solutions "shop in shop" comparables dans d'autres secteurs de l'économie. Nous voudrions reprendre cette théorie et montrer, à l'exemple de la Klinik Hirslanden de Zurich (ci-après Clinique Hirslanden), qu'une organisation différenciée spécifique aux fonctions du corps médical offre de solides avantages aux patients, médecins, hôpitaux et organismes payeurs.

\section{Hirslanden: fusion des systèmes de médecin-chef et de médecins accrédités}

Ces dix dernières années, la direction de la clinique et le corps médical de la Clinique Hirslanden ont transformé cette dernière, la faisant passer d'une infrastruc- 
ture médico-technique mise à disposition de médecins indépendants travaillant généralement seuls, en un système intégré de soins stationnaires organisés autour de centres accrédités. Cela permet à la Clinique Hirslanden de proposer aujourd'hui, en tant qu'établissement listé, l'ensemble du spectre des prestations d'un hôpital général. Un facteur de succès fondamental a été en ce sens l'organisation différenciée du corps médical en un "système Hirslanden", dans lequel les systèmes de médecin-chef et de médecins accrédités ont été fusionnés. Ce système définit le rôle et l'organisation des médecins de manière différente pour les prestations médicales de base que pour les prestations spécialisées - et incarne ainsi, en un sens, le concept de groupes de prestations de la planification hospitalière du canton de Zurich.

Le socle de la liste des hôpitaux forment, avec la médecine d'urgence, l'anesthésie, la médecine intensive, la médecine générale, la chirurgie générale et la radiologie, les soins de base stationnaires. Il ne faut pas se méprendre sur la notion de «soins de base». Elle désigne la fonction d'une prestation dans l'hôpital, et non son degré de complexité. Cela veut dire que des prestations de médecine intensive typiquement perçues comme étant (hautement) spécialisées du point de vue médical sont malgré tout classées dans les soins de base internes à l'hôpital du point de vue fonctionnel.

Dans le modèle Hirslanden, les services médicaux et non médicaux des soins de base internes à l'hôpital sont rassemblés dans l'unité organisationnelle "système médical». Cette dernière est dirigée par un médecin exempt de tâches cliniques, qui gère en tant que responsable à temps complet les processus médicaux-clés. Les médecins du système médical sont employés et soumis à la direction d'un médecin-chef, comme les thérapeutes et soignants par ailleurs. Le système médical pousse à une forte intégration des services médicaux et paramédicaux, et ainsi à une haute intégration interdisciplinaire des processus de prise en charge.

D’un autre côté, les centres médicaux (centre de cardiologie, centre du pied, etc.) fournissent - en collaboration avec le système médical - des prestations spécialisées et hautement spécialisées. Les centres sont gérés la plupart du temps sous forme de cabinets de groupes mono-disciplinaires de médecins accrédités: ils louent des locaux dans l'hôpital, sont dirigés collégialement et leurs formes juridiques et organisationnelles diffèrent (par ex. cabinet collectif, modèle de partenariat analogue aux cabinets d'avocat, ou société anonyme avec médecins salariés). Les centres in-house sont étroitement liés à l'hôpital par des contrats transpa- rents et une organisation solide des processus; ils s'engagent entre autre à participer au service des urgences de leur spécialité médicale, à respecter les normes de qualité de l'hôpital et à définir des normes de traitement uniformes à l'intérieur du centre.

Les médecins du système médical mettent ainsi leurs prestations à disposition des patients et des médecins des centres médicaux en tant qu'une offre intégrée de soins de base; ils gèrent les comorbidités et les complications et permettent ainsi aux médecins des centres de se concentrer sur leurs activités spécialisées principales. De leur côté, les médecins des centres doivent maintenir le positionnement sur le marché de leurs centres grâce à de bons résultats cliniques et des relations privilégiés avec leurs pourvoyeurs. La fourniture de prestations est ainsi divisée est dirigée par le service de planification des lits et du bloc opératoire, qui est lui-même directement subordonné à la direction de l'hôpital et par le service qualité clinique de l'hôpital (coordination des standards de prise en charge, colloques pluridisciplinaires d'indications, commissions mortalité \& morbidité, CIRS, direction des registres, hygiène hospitalière, etc.).

\section{Un médecin exempt de tâches cliniques dirige} l'unité organisationnelle «système médical» en tant que responsable des processus médicaux-clés.

Cette organisation duale du corps médical (médecins de premier recours gérés dans le système dit de chef de service et centres spécialisés de médecins accrédités), complétée par une gestion centralisée de la qualité et des performances, a si bien fait ses preuves pour le corps médical et la direction de la Clinique Hirslanden que le modèle est devenu un exemple pour l'ensemble du groupe Hirslanden.

\section{Les éléments du modèle intégré}

Parce que nous sommes convaincus que ce modèle peut également aider d'autres hôpitaux, quelle que soit leur forme juridique ou leur responsabilité, à affronter les défis du futur, nous voudrions proposer à la discussion un modèle intégré dont les bases sont constituées des quatre éléments suivants:

\section{Unités de services médicaux}

Les unités de services médicaux (fourniture de prestations; en vert dans fig. 1) sont formées de médecins qui assument la fonction de généralistes pour l'hôpital et de prestataires pour les patients et les spécialistes. Elles comprennent des unités de services médicaux (anesthésie, médecine intensive, radiologie, médecine 
d'urgence, etc.) et non médicales (soins, thérapies, etc.) et elles relèvent d'une direction commune. Elles régissent l'infrastructure hospitalière médicale centrale, comme le service des urgences, le bloc opératoire, l'unité de soins intensifs et les étages de lits. Elles assurent également le service des urgences pour les soins de base. Leur principal objectif est une forte intégration interdisciplinaire de tous les processus de traitements stationnaires ainsi que la sécurité des patients. En étroite concertation avec les unités médicales spécialisées, les unités de services médicaux sont impliquées dans toutes les étapes du processus de traitement des patients, de l'entrée à la sortie de l'hôpital. Les médecins de ces unités dépendent de l'hôpital et sont gérés dans le système de chef de service. Ces médecins-chefs dépendent directement du directeur médical des unités de services médicaux, qui doit être membre de la direction de l'hôpital.

\section{Unités médicales spécialisées}

Les unités médicales spécialisées (fourniture de prestations; en bleu foncé dans fig. 1) assument les sousspécialités (par ex. gastro-entérologie, chirurgie de la colonne vertébrale). Elles endossent la responsabilité entrepreneuriale pour la fourniture des prestations médicales spécialisées, les relations avec les pourvoyeurs, l'acquisition de patientèle ainsi que l'innovation dans leurs spécialités; elles assurent, en coopération avec les unités de services médicaux, le service des urgences pour la spécialité médicale concernée. Les médecins des unités spécialisées sont soit engagés par l'hôpital ou sont accrédités et peuvent s'organiser de diverses manières (par ex. dans un système de présidence, un système de partenaires ou encore un système de médecin-chef) mais ils doivent démontrer une bonne cohérence de groupe.

\section{Gestion de la qualité et des performances médicales}

La gestion de la qualité et des performances médicales (unité pour la conduite de la fourniture des prestations; en bleu clair sur fig. 1). Elle assure, par une planification centralisée, la gestion des plages opératoires, des lits ainsi que la gouvernance centralisée de la qualité clinique et de l'économicité de l'hôpital. Elle soulage ainsi les unités de services médicaux et les unités spécialisées des tâches de planification et d'administration. Dans un objectif de séparation des pouvoirs

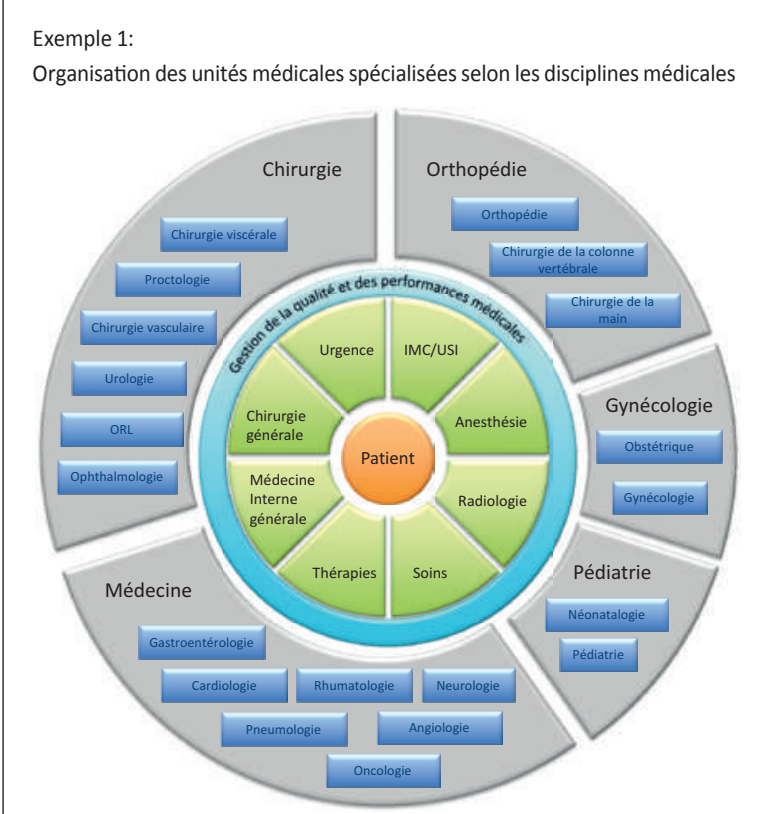

Exemple 2:

Organisation des unités médicales spécialisées selon les systèmes organes
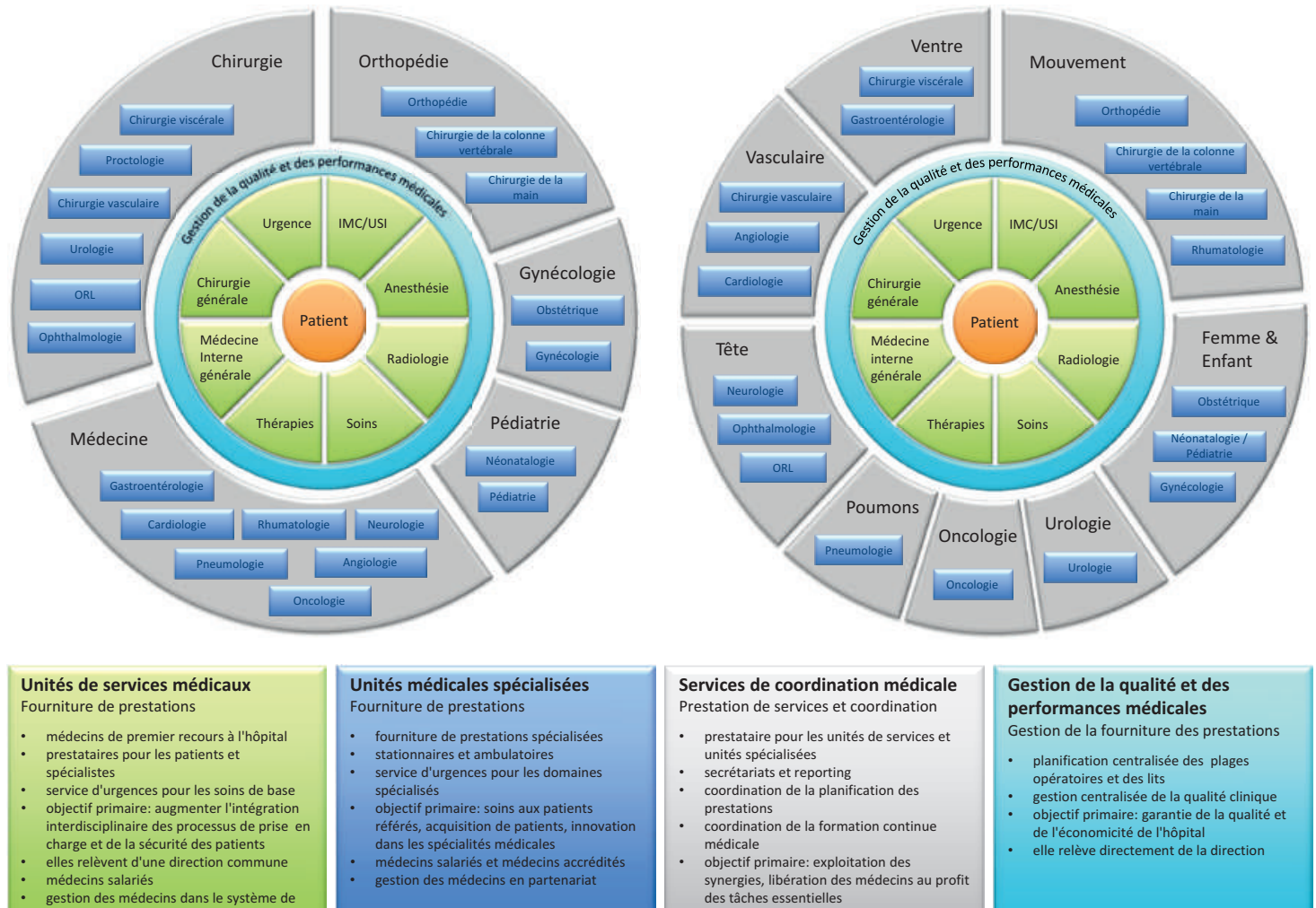

Figure 1: Possibilités d'organisation du modèle de système intégré dans les hôpitaux de soins aigus (sélection des unités spécialisées est simplement un exemple). 
effective et indépendante des intérêts, cette unité ne doit pas dépendre des cadres dirigeants des unités de services médicaux ou des unités spécialisées, mais doit plutôt être dirigée par des médecins dépendant directement de la direction de l'hôpital.

\section{Unités de coordination médicale}

Les unités de coordination médicale (unités de prestations; en gris dans fig. 1). Elles prennent en charge les tâches de coordination et d'administration telles que la planification des piquets, le reporting, l'organisation de la formation continue médicale, la coordination des standards de prise en charge entre spécialités médicales etc. pour un groupe d'unités de services médicaux et d'unités spécialisées médicales apparentées. Elles tirent ainsi profit de synergies et génèrent un gain d'efficience dans les services non cliniques, ce qui laisse plus de place pour les tâches médicales essentielles.

Par rapport aux systèmes de gestion existant dans de nombreux hôpitaux, notre modèle accorde une plus grande importance à la répartition des tâches entre professions et unités opérationnelles, ainsi qu'à une organisation interne adaptée spécifiquement aux unités opérationnelles et à la gestion des médecins.

Du fait des conditions-cadres variées des hôpitaux suisses, notre modèle se limite délibérément à quelques principes. La mise en œuvre de l'organisation, de la direction et des aspects contractuels doit ainsi être adaptée aux différents sites. Le Groupe de cliniques privées Hirslanden lui-même ne met pas en œuvre tous les éléments du modèle dans l'ensemble de ses cliniques. Nous sommes toutefois convaincus que les éléments du modèle décrit ont une universalité certaine et présentent un grand potentiel pour la gestion moderne des hôpitaux.

En ce sens, l'exemple de la figure 1 est à comprendre comme un schéma idéal, à adapter individuellement pour chaque hôpital.

\section{Le modèle offre du potentiel significatif pour le corps médical}

Le modèle offre tout particulièrement pour le corps médical un potentiel significatif:

- pour les médecins cadres en général: gain d'efficience sur les tâches non cliniques et donc gain de temps pour les tâches essentielles;

Dr Daniel Liedtk

Groupe de cliniques privées Hirslanden

Seefeldstrasse 214

CH-8008 Zurich services et de l'efficience des processus, grâce à une intégration renforcée et une gestion uniforme des soins médicaux de base dans les unités de services médicaux;

- gestion des ressources centralisée par hôpital et donc plus efficiente (planification des plages opératoires et des lits);

- gestion de la qualité centralisée et donc fiabilité de son contrôle et de son amélioration;

- exploitation du potentiel entrepreneurial des spécialistes dans les unités spécialisées;

- intégration facilitée des médecins accrédités dans toutes les sous-spécialités (pas uniquement dans les petites branches);

- simplification de la répartition du travail entre spécialistes, ainsi que du travail des spécialistes à temps partiel entre plusieurs hôpitaux et donc désamorçage de la pénurie en personnel qualifié;

- augmentation de l'attractivité de l'hôpital pour les spécialistes hautement qualifiés grâce à des solutions d'emploi flexibles et à une plus grande liberté d'entreprise;

- réduction de la distance entre médecins hospitaliers travaillant principalement en domaine stationnaire et médecins spécialisés travaillant de manière indépendante et principalement en domaine ambulatoire - le modèle est une réponse moderne au virage ambulatoire de la médecine.

Le modèle implique un changement d'approche. Le «département» médical classique, organisé hiérarchiquement, doit devenir une petite unité de coordination sans pouvoir de planification sur les lits ou les disponibilités opératoires. La fonction de "médecin-chef» doit être recentrée, en premier lieu, sur les tâches cliniques essentielles du médecin, la formation continue médicale et l'innovation dans les branches spécialisées. Les médecins accrédités doivent se former de manière croissante en unités spécialisées organisées collégialement et coopérer étroitement avec les unités de services médicaux. Cela ne se fera pas en un jour, mais cela en vaut vraiment la peine.

Nous présenterons une sélection d'éléments et fonctions du modèle de système intégré dans un article de la prochaine édition du Bulletin des médecins suisses.

\section{Références}

1 Michael E. Porter, Elizabeth O. Teisberg. Redefining Health Care: Creating Value-Based Competition on Results. 2006.

2 Les hôpitaux gaspillent des milliards. SonntagsBlick du 22 février 2015.

- Littérature disponible chez Daniel Liedtke.
Remerciement

Nous remercions Cédric Bossart pour la traduction de ces articles. 